PROCEEDINGS OF THE

AMERICAN MATHEMATICAL SOCIETY

Volume 133, Number 1, Pages 57-63

S 0002-9939(04)07557-4

Article electronically published on June 18, 2004

\title{
BETTI NUMBERS OF SOME MONOMIAL IDEALS
}

\author{
GIUSEPPE VALLA
}

(Communicated by Bernd Ulrich)

\begin{abstract}
In this paper we compute the graded Betti numbers of certain monomial ideals that are not stable. As a consequence we prove a conjecture, stated by G. Fatabbi, on the graded Betti numbers of two general fat points in $\mathbf{P}^{3}$.
\end{abstract}

\section{INTRODUCTION}

Let $R:=k\left[X_{1}, \ldots, X_{n}\right]$ be a polynomial ring in $n$ variables over a field $k$. Given a graded module $M=\bigoplus_{t} M_{t}$ over $R$, its minimal graded free resolution will be written as

$$
0 \rightarrow \bigoplus_{j} R(-j)^{\beta_{h, j}} \rightarrow \cdots \rightarrow \bigoplus_{j} R(-j)^{\beta_{1, j}} \rightarrow \bigoplus_{j} R(-j)^{\beta_{0, j}} \rightarrow M \rightarrow 0 .
$$

The integers $\beta_{i, j}$ are the graded Betti numbers of $M$. A particularly simple sort of graded module is provided by the ideals generated by monomials, which we call monomial ideals. However, despite the misleading appearence of simplicity, it is still an open problem to describe explicitly the graded Betti numbers even in this case. (See 2], p. 29.)

In [6] D. Taylor has given a resolution of monomial ideals which, in general, is not minimal. Neither is the more recent and improved version given by G. Lyubeznik in [5] minimal. An explicit minimal resolution for a family of monomial ideals, which are called stable ideals, has been given by S. Eliahou and M. Kervaire in [3].

Let us recall here that if $w$ is a monomial in $R$, we let $\max (w)$ denote the largest index of the variables dividing $w$. A monomial ideal $I$ in $R$ will be said to be stable if for every monomial $w \in I$ and index $i<m=\max (w)$, the monomial $x_{i} w / x_{m}$ again belongs to $I$.

In the nonstable case, the computation of the graded Betti numbers of a monomial ideal is always very hard.

In this paper we compute the graded Betti numbers for a class of monomial ideals that are not stable, by using the notion of "splitting" of a monomial ideal introduced by Eliahou and Kervaire in their paper.

This particular family of ideals is interesting because each ideal in the family defines an algebra that is the artinian reduction of the homogeneous coordinate

Received by the editors May 12, 2003 and, in revised form, October 13, 2003.

2000 Mathematics Subject Classification. Primary 13D40; Secondary 13P99.

Key words and phrases. Monomial ideal, Betti number, ideal of fat points.

(C)2004 American Mathematical Society 
ring of the scheme of two general fat points in $\mathbf{P}^{n}$. Hence we can prove very precise assertions about Betti numbers of such kinds of projective schemes.

In the case $n=3$, this gives a positive answer to a conjecture stated by G. Fatabbi in [4]. This paper was in fact motivated by an attempt to understand this conjecture.

\section{The FIRST AND the LAST Betti NUMBeR}

The monomial ideals we want to study are easily defined as follows. Let $a$ and $b$ be positive integers such that $a \geq b$ and let $J:=\left(X_{2}, \ldots, X_{n}\right)$. Then we define $I_{a, b}$ to be the ideal generated by

$$
\begin{aligned}
\left(X_{1}^{a+b}, X_{1}^{a+b-2} J, X_{1}^{a+b-4} J^{2}, \ldots, X_{1}^{a-b+2} J^{b-1},\right. \\
\\
\left.\quad X_{1}^{a-b} J^{b}, X_{1}^{a-b-1} J^{b+1}, \ldots, X_{1} J^{a-1}, J^{a}\right)
\end{aligned}
$$

where $X_{1}^{t} J^{s}$ means the set of monomials obtained by multiplying $X_{1}^{t}$ by all the monomials that are the standard minimal generators of $J^{s}$.

For short, we can write

$$
I_{a, b}=\left(X_{1}^{a+b-2 j} J^{j}, X_{1}^{a-t} J^{t}\right)_{\substack{j=0, \ldots, b-1 \\ t=b, \ldots, a}} .
$$

It is clear that $I_{a, b}$ is not stable since for example if $a=2$ and $b=1$ we have

$$
I_{2,1}=\left(X_{1}^{3}, X_{1} X_{2}, X_{1} X_{3}, X_{2}^{2}, X_{2} X_{3}, X_{3}^{2}\right)
$$

so that $X_{1} X_{2} \in I_{2,1}$ but $X_{1}^{2} \notin I_{2,1}$.

Since we know a minimal set of generators for $I_{a, b}$, we immediately get the first graded Betti numbers of $R / I_{a, b}$. Namely, the monomials $\left\{X_{1}^{a-t} J^{t}\right\}$ have degree $a$ and their number is $\left(\begin{array}{c}(n-1)+t-1 \\ t\end{array}\right)$. Thus for every $t=b, \ldots, a$ hence, all together, we have

$$
\sum_{t=b}^{a}\left(\begin{array}{c}
n+t-2 \\
t
\end{array}\right)=\left(\begin{array}{c}
n+a-1 \\
a
\end{array}\right)-\left(\begin{array}{c}
n+b-2 \\
b-1
\end{array}\right)
$$

generators of degree $a$.

The monomials $\left\{X_{1}^{a+b-2 s} J^{s}\right\}$ have degree $a+b-s$ and their number is $\left(\begin{array}{c}(n-1)+s-1 \\ s\end{array}\right)$ for every $s=0, \ldots, b-1$. This proves that

$$
\beta_{1, j}\left(R / I_{a, b}\right)= \begin{cases}\left(\begin{array}{c}
n+a-1 \\
a
\end{array}\right)-\left(\begin{array}{c}
n+b-2 \\
b-1
\end{array}\right) & \text { if } j=a \\
\left(\begin{array}{c}
n+a+b-j-2 \\
a+b-j
\end{array}\right) & \text { if } j=a+1, \ldots, a+b, \\
0 & \text { if } j \geq a+b+1 .\end{cases}
$$

The ideal $I_{a, b}$ has a nice property. Namely, we can easily compute the socle of $R / I_{a, b}$ because of the following result.

Lemma 2.1. Let $I_{a, b}$ be as above. Then

$$
I_{a, b}:\left(X_{1}, \ldots, X_{n}\right)=I_{a, b}:\left(X_{1}\right)=\left(X_{1}^{a+b-2 j-1} J^{j}, X_{1}^{a-t-1} J^{t}\right)_{\substack{j=0, \ldots, b-1 \\ t=b, \ldots, a-1}}
$$

Proof. We only need to prove that $I_{a, b}:\left(X_{1}\right)=I_{a, b}:\left(X_{1}, \ldots, X_{n}\right)$ since the other equality is clear. We have $I_{a, b}:\left(X_{1}, \ldots, X_{n}\right) \subseteq I_{a, b}:\left(X_{1}\right)$. On the other hand, since $I_{a, b}$ is a monomial ideal, if $F X_{1} \in I_{a, b}$, then all the monomials of $F X_{1}$ are in $I_{a, b}$. In other words $m X_{1} \in I_{a, b}$ for all monomials $m$ of $F$. This means that either $m X_{1}=X_{1}^{a+b-2 j} H$ for some $j=0, \ldots, b-1$, and some monomial $H$ of degree bigger than or equal to $j$, or $a>b$ and $m X_{1}=X_{1}^{a-t} H$ for some $b \leq t<a$ 
and some monomial $H$ of degree bigger than or equal to $t$. In the first case we get $m=X_{1}^{a+b-2 j-1} H$ so that, if $j \geq 2$,

$$
m X_{i}=X_{1}\left[X_{1}^{a+b-2(j+1)}\left(X_{i} H\right)\right] \subseteq\left(X_{1}^{a+b-2(j+1)} J^{j+1}\right) \subseteq I_{a, b},
$$

in the second case $m=X_{1}^{a-t-1} H$ so that, if $j \geq 2$,

$$
m X_{i}=X_{1}^{a-(t+1)}\left(X_{i} H\right) \subseteq\left(X_{1}^{a-(t+1)} J^{t+1}\right) \subseteq I_{a, b},
$$

and the conclusion follows.

Since the last shifts in the resolution are the degrees of the generators of the socle increased by $n$, we get:

Corollary 2.2. Let $I_{a, b}$ be as above. Then we have

$$
\beta_{n, j}\left(R / I_{a, b}\right)= \begin{cases}\sum_{t=b}^{a-1}\left(\begin{array}{c}
n+t-2 \\
t
\end{array}\right) & \\
=\left(\begin{array}{c}
n+a-2 \\
a-1
\end{array}\right)-\left(\begin{array}{c}
n+b-2 \\
b-1
\end{array}\right) & \text { if } j=a+n-1, \\
\left(\begin{array}{c}
2 n+a+b-j-3 \\
n+a+b-j-1
\end{array}\right) & \text { if } j=a+n, \ldots, a+b+n-1, \\
0 & \text { if } j \geq a+b+n .\end{cases}
$$

We may also easily compute the Hilbert function of $R / I_{a, b}$.

Namely, we only need to remark that for $a \leq t \leq a+b-1$ we have

$$
X_{1}^{2 t-b-a} J^{b+a-t}=X_{1}^{a+b-2(a+b-t)} J^{b+a-t} \in I_{a, b},
$$

so that the monomials of degree $t$ that are not in $I_{a, b}$ are exactly the monomials $\left\{X_{1}^{t}, X_{1}^{t-1} J, X_{1}^{t-2} J^{2}, \ldots, X_{1}^{2 t-b-a+1} J^{b+a-t-1}\right\}$. The number of these monomials is

$$
1+(n-1)+\left(\begin{array}{l}
n \\
2
\end{array}\right)+\cdots+\left(\begin{array}{c}
n-1+b+a-t-1-1 \\
b+a-t-1
\end{array}\right)=\left(\begin{array}{c}
n+b+a-t-2 \\
n-1
\end{array}\right) .
$$

Hence we get the following formula.

Proposition 2.3. Let $I_{a, b}$ be as above. Then

$$
H_{R / I_{a, b}}(t)= \begin{cases}\left(\begin{array}{c}
n+t-1 \\
t
\end{array}\right) & \text { if } t<a \\
\left(\begin{array}{c}
n+b+a-t-2 \\
n-1
\end{array}\right) & \text { if } a \leq t \leq a+b-1, \\
0 & \text { if } t \geq a+b .\end{cases}
$$

In the case $n=3$ we know that the Hilbert series of $R / I_{a, b}$ is given by

$$
P_{R / I_{a, b}}(z):=\sum_{t} H_{R / I_{a, b}}(t) z^{t}=\frac{1-\sum_{j} \beta_{1, j} z^{j}+\sum_{j} \beta_{2, j} z^{j}-\sum_{j} \beta_{3, j} z^{j}}{(1-z)^{3}} .
$$

Hence we immediately get all the Betti numbers in the resolution.

Namely, it is easy to see that

$$
\begin{aligned}
(1-z)^{3} P_{R / I_{a, b}}(z) & \\
= & 1+\left[\left(\begin{array}{c}
b+1 \\
2
\end{array}\right)-\left(\begin{array}{c}
a+2 \\
2
\end{array}\right)\right] z^{a}+[a(a+2)-b(b+2)] z^{a+1} \\
& +\left[\left(\begin{array}{c}
b+2 \\
2
\end{array}\right)-\left(\begin{array}{c}
a+1 \\
2
\end{array}\right)\right] z^{a+2},
\end{aligned}
$$

so that, using the above formula, we get the following proposition. 
Proposition 2.4. Let $n=3$ and $I_{a, b}$ be as above. Then we have

$$
\beta_{2, j}\left(R / I_{a, b}\right)= \begin{cases}a(a+2)-b(b+1) & \text { if } j=a+1 \\ 2(a+b-j+2) & \text { if } j=a+2, \ldots, a+b+1, \\ 0 & \text { if } j \geq a+b+2 .\end{cases}
$$

\section{The MAIN RESUlT}

In this section we compute the intermediate graded Betti numbers of the ideal $R / I_{a, b}$. Let us start with the case $b=a$, which is easy and the right initial step in the induction argument for the general case.

So let $I_{a}:=I_{a, a}=\left(X_{1}^{2(a-j)} J^{j}, J^{a}\right)$ with $j=0, \ldots, a-1$. We first remark that if $a \geq 2$, then we have the decomposition

$$
I_{a}=X_{1}^{2} I_{a-1}+J^{a} .
$$

This decomposition is a splitting of the monomial ideal $I_{a}$. Here we recall the definition of splittable ideal given in [3], page 17. For a monomial ideal $I$, we shall denote by $G(I)$ the canonical generator system of $I$, which is the set of all monomials in $I$ that are not proper multiples of any monomial in $I$.

Definition 3.1. We say that a monomial ideal $I$ is splittable if $I$ is the sum of two nonzero monomial ideals $U$ and $V$ such that:

1. $G(I)$ is the disjoint union of $G(U)$ and $G(V)$.

2. There is a splitting function

$$
\begin{gathered}
G(U \cap V) \rightarrow G(U) \times G(V), \\
w \rightarrow(\phi(w), \psi(w))
\end{gathered}
$$

satisfying the following properties:

a) $w=\operatorname{lcm}(\psi(w), \phi(w))$ for all $w \in G(W):=G(U \cap V)$.

b) For every subset $G^{\prime} \subseteq G(W)$, both $l \mathrm{~cm}\left(\phi\left(G^{\prime}\right)\right)$ and $l \mathrm{~cm}\left(\psi\left(G^{\prime}\right)\right)$ strictly divide $\operatorname{lcm}\left(G^{\prime}\right)$.

This notion is relevant for our problem because of the following result.

Proposition 3.2. Let $I$ be a splittable monomial ideal with splitting $I=U+V$. Then for all $q \geq 1$ and for all $j$ we have

$$
\beta_{q, j}(R / I)=\beta_{q, j}(R / U)+\beta_{q, j}(R / V)+\beta_{q-1, j}(R / U \cap V) .
$$

A proof of this result can be found in [3] where only the global Betti numbers are considered. But, as remarked in [4], the same proof works for the graded Betti numbers as well.

In the following we need to recall that $J^{t}$ has a linear resolution so that

$$
\left\{\begin{array}{l}
\beta_{i, j}\left(R / J^{t}\right)=0 \\
\beta_{i, t+i-1}\left(R / J^{t}\right)=\left(\begin{array}{c}
n+t-2 \\
t+i-1
\end{array}\right)\left(\begin{array}{c}
t+i-2 \\
i-1
\end{array}\right)
\end{array} \text { if } j \neq t+i-1,\right.
$$

(see, for example, [1], Proposition 1.6).

We will simply write $\beta_{i}\left(R / J^{t}\right)$ instead of $\beta_{i, t+i-1}\left(R / J^{t}\right)$. 
Theorem 3.3. Let $I_{a}$ be as above. Then we have

$$
\beta_{i, j}\left(R / I_{a}\right)= \begin{cases}\beta_{i}\left(R / J^{a}\right)=\left(\begin{array}{c}
n+a-2 \\
a+i-1
\end{array}\right)\left(\begin{array}{c}
a+i-2 \\
i-1
\end{array}\right) & \text { if } j=a+i-1, \\
\left(\begin{array}{l}
n-1 \\
i-1
\end{array}\right)\left(\begin{array}{c}
n+2 a-j+i-3 \\
n-2
\end{array}\right) & \text { if } a+i \leq j \leq 2 a+i-1, \\
0 & \text { if } j \geq 2 a+i .\end{cases}
$$

Proof. As we have already seen, if $a \geq 2$, we have $I_{a}=X_{1}^{2} I_{a-1}+J^{a}$. We prove that this is a splitting for the ideal $I_{a}$. Since $X_{1}$ is a regular element modulo $J$, we have

$$
\left(X_{1}^{2} I_{a-1}\right) \cap J^{a}=X_{1}^{2}\left(I_{a-1} \cap J^{a}\right)=X_{1}^{2} J^{a} .
$$

Define the splitting function as follows:

$$
\begin{gathered}
G\left(X_{1}^{2} J^{a}\right) \rightarrow G\left(X_{1}^{2} I_{a-1}\right) \times G\left(J^{a}\right), \\
X_{1}^{2} w \rightsquigarrow\left(X_{1}^{2} \widetilde{w}, w\right)
\end{gathered}
$$

where $\widetilde{w}$ is the monomial obtained by decreasing by one the first nonzero exponent in $w$. It is clear that $\operatorname{lcm}\left(X_{1}^{2} \widetilde{w}, w\right)=X_{1}^{2} w$. On the other hand, if $G^{\prime}=$ $\left\{X_{1}^{2} F_{1}, \ldots, X_{1}^{2} F_{r}\right\}$ is a subset of $G\left(X_{1}^{2} J^{a}\right)$, then $\operatorname{lcm}\left(G^{\prime}\right)=X_{1}^{2} \operatorname{lcm}\left(\widetilde{F_{1}}, \ldots, \widetilde{F_{r}}\right)$, and $\operatorname{lcm}\left(F_{1}, \ldots, F_{r}\right)$ strictly divide $\operatorname{lcm}\left(G^{\prime}\right)=X_{1}^{2} \operatorname{lcm}\left(F_{1}, \ldots, F_{r}\right)$.

It follows that

$$
\beta_{i, j}\left(R / I_{a}\right)=\beta_{i, j-2}\left(R / I_{a-1}\right)+\beta_{i, j}\left(R / J^{a}\right)+\beta_{i-1, j-2}\left(R / J^{a}\right) .
$$

Let $j=a+i-1$; we have

$$
\beta_{i, a+i-1}\left(R / I_{a}\right)=\beta_{i, a+i-3}\left(R / I_{a-1}\right)+\beta_{i, a+i-1}\left(R / J^{a}\right)+\beta_{i-1, a+i-3}\left(R / J^{a}\right) .
$$

Since the initial degree of $I_{a-1}$ is $a-1$ and that of $J^{a}$ is $a$, the first and the last summand in the above formula are zero so that the conclusion follows in this case.

The theorem being trivial in the case $a=1$, we may argue by induction on $a$. Let $j \geq a+i+1$; we have

$$
\beta_{i, j}\left(R / I_{a}\right)=\beta_{i, j-2}\left(R / I_{a-1}\right)+\beta_{i, j}\left(R / J^{a}\right)+\beta_{i-1, j-2}\left(R / J^{a}\right) .
$$

In this case the second and the last summand are zero, so that, since $j-2 \geq$ $(a-1)+i$, we apply the inductive assumption and we get

$$
\begin{gathered}
\beta_{i, j}\left(R / I_{a}\right)=\beta_{i, j-2}\left(R / I_{a-1}\right)=\left(\begin{array}{c}
n-1 \\
i-1
\end{array}\right)\left(\begin{array}{c}
n+2(a-1)-(j-2)+i-3 \\
n-2
\end{array}\right) \\
=\left(\begin{array}{c}
n-1 \\
i-1
\end{array}\right)\left(\begin{array}{c}
n+2 a-j+i-3 \\
n-2
\end{array}\right) .
\end{gathered}
$$

Finally, let $j=a+i$. In this case in the above formula only the summand in the middle is zero and so we get

$$
\begin{gathered}
\beta_{i, a+i}\left(R / I_{a}\right)=\beta_{i, a+i-2}\left(R / I_{a-1}\right)+\beta_{i-1, a+i-2}\left(R / J^{a}\right) \\
=\left(\begin{array}{c}
n+a-3 \\
a+i-2
\end{array}\right)\left(\begin{array}{c}
a+i-3 \\
i-1
\end{array}\right)+\left(\begin{array}{c}
a+n-2 \\
a+i-2
\end{array}\right)\left(\begin{array}{c}
a+i-3 \\
i-2
\end{array}\right)=\left(\begin{array}{c}
n-1 \\
i-1
\end{array}\right)\left(\begin{array}{c}
n+a-3 \\
n-2
\end{array}\right)
\end{gathered}
$$

where the last equality is an easy computation. This proves the theorem. 
We come now to the general case.

Theorem 3.4. Let $I_{a, b}$ be as above. Then we have

$$
\beta_{i, j}\left(R / I_{b, a}\right)= \begin{cases}\sum_{r=b}^{a} \beta_{i}\left(R / J^{r}\right) & \\
+\sum_{r=b+1}^{a} \beta_{i-1}\left(R / J^{r}\right) & \text { if } j=a+i-1, \\
\left(\begin{array}{c}
n-1 \\
i-1
\end{array}\right)\left(\begin{array}{c}
n+a+b-j+i-3 \\
n-2
\end{array}\right) & \text { if } a+i \leq j \leq a+b+i-1, \\
0 & \text { if } j \geq a+b+i .\end{cases}
$$

Proof. Let us assume $a>b$. Then we have

$$
I_{b, a}=X_{1} I_{b, a-1}+J^{a}
$$

and the intersection of the two ideals is $X_{1} J^{a}$. As before it is easy to see that this is a splitting so that we have

$$
\beta_{i, j}\left(R / I_{b, a}\right)=\beta_{i, j-1}\left(R / I_{b, a-1}\right)+\beta_{i, j}\left(R / J^{a}\right)+\beta_{i-1, j-1}\left(R / J^{a}\right) .
$$

Let us first assume that $j \geq a+i$. In the above formula, the last two addenda are zero so that we get

$$
\begin{gathered}
\beta_{i, j}\left(R / I_{b, a}\right)=\beta_{i, j-a+b}\left(R / I_{b}\right)=\left(\begin{array}{c}
n-1 \\
i-1
\end{array}\right)\left(\begin{array}{c}
n+2 b-(j-a+b)+i-3 \\
n-2
\end{array}\right), \\
=\left(\begin{array}{c}
n-1 \\
i-1
\end{array}\right)\left(\begin{array}{c}
n+a+b-j+i-3 \\
n-2
\end{array}\right),
\end{gathered}
$$

as wanted.

Finally, let $j=a+i-1$. In this case the formula is

$$
\beta_{i, a+i-1}\left(R / I_{b, a}\right)=\beta_{i, a+i-2}\left(R / I_{b, a-1}\right)+\beta_{i, a+i-1}\left(R / J^{a}\right)+\beta_{i-1, a+i-2}\left(R / J^{a}\right)
$$

and no summand is zero; this is also the case when we compute $\beta_{i, a+i-2}\left(R / I_{b, a-1}\right)$, and so on. The conclusion follows immediately.

As an application let us compute again $\beta_{2, a+1}\left(R / I_{b, a}\right)$ in the case $n=3$. We have

$$
\beta_{2, a+1}\left(R / I_{b, a}\right)=\sum_{r=b}^{a} \beta_{i}\left(R / J^{r}\right)+\sum_{r=b+1}^{a} \beta_{i-1}\left(R / J^{r}\right) .
$$

But $J=\left(X_{2}, X_{3}\right)$ so that

$$
\beta_{1}\left(R / J^{t}\right)=t+1, \quad \beta_{2}\left(R / J^{t}\right)=t .
$$

This implies

$$
\begin{aligned}
& \beta_{2, a+1}\left(R / I_{b, a}\right)=b+(b+1)+\cdots+a+(b+2)+\cdots+(a+1) \\
& =\left(\begin{array}{c}
a+1 \\
2
\end{array}\right)-\left(\begin{array}{l}
b \\
2
\end{array}\right)+\left(\begin{array}{c}
a+2 \\
2
\end{array}\right)-\left(\begin{array}{c}
b+2 \\
2
\end{array}\right)=a(a+2)-b(b+1) .
\end{aligned}
$$

Finally, let $j \geq a+2$; we have

$$
\beta_{2, j}\left(R / I_{b, a}\right)=2(a+b-j+2) .
$$

These are the values already found at the end of section 2 .

We end the paper by remarking that, as a direct application of these results, we are able to compute the graded Betti numbers of two general fat points in $\mathbf{P}^{n}$. In the particular case when $n=3$, this proves Conjecture 1 in [4]. 
Let $X=\left\{\left(P_{1}, a\right),\left(P_{2}, b\right)\right\}$ with $a \geq b$ a set of two general fat points in $\mathbf{P}^{n}$. We may assume that $P_{1}=(1,0,0, \ldots, 0)$ and $P_{2}=(0,1,0, \ldots, 0)$ and as before let $J=\left(X_{2}, \ldots, X_{n}\right)$. The defining ideal $I(X)$ of $X$ is

$$
\begin{gathered}
\left(X_{1}, \ldots, X_{n}\right)^{a} \cap\left(X_{0}, X_{2}, \ldots, X_{n}\right)^{b}= \\
\left(X_{0}^{b} X_{1}^{a}, X_{0}^{b-1} X_{1}^{a-1} J, \ldots, X_{0} X_{1}^{a-b+1} J^{b-1}, X_{1}^{a-b} J^{b}, X_{1}^{a-b-1} J^{b+1}, \ldots, X_{1} J^{a-1}, J^{a}\right) .
\end{gathered}
$$

Now it is clear that $X_{0}-X_{1}$ is not a zero-divisor modulo this ideal, so that the graded Betti numbers of $k\left[X_{0}, \ldots, X_{n}\right] / I(X)$ coincide with the graded Betti numbers of $k\left[X_{0}, \ldots, X_{n}\right] / I(X)+\left(X_{0}-X_{1}\right)$. Since we have

$$
k\left[X_{0}, \ldots, X_{n}\right] / I(X)+\left(X_{0}-X_{1}\right) \simeq k\left[X_{1}, \ldots, X_{n}\right] / I_{a, b},
$$

we get the following corollary:

Corollary 3.5. Let $X=\left\{\left(P_{1}, a\right),\left(P_{2}, b\right)\right\}$ with $a \geq b$ be a set of two general fat points in $\mathbf{P}^{n}$. Then

$$
\beta_{i, j}(X)= \begin{cases}\sum_{r=b}^{a} \beta_{i}\left(R / J^{r}\right)+\sum_{r=b+1}^{a} \beta_{i-1}\left(R / J^{r}\right) & \text { if } j=a+i-1, \\
\left(\begin{array}{c}
n-1 \\
i-1
\end{array}\right)\left(\begin{array}{c}
n+a+b-j+i-3 \\
n-2
\end{array}\right) & \text { if } a+i \leq j \leq a+b+i-1, \\
0 & \text { if } j \geq a+b+i .\end{cases}
$$

\section{REFERENCES}

[1] Cavaliere M.P., Rossi M.E., Valla G., On the resolution of certain graded algebras, Trans. A.M.S. 337 (1983), 389-409. MR93m:13004

[2] De Concini C., Eisenbud D., Procesi C., Hodge Algebras, Astérisque 91 (1982). MR85d:13009

[3] Eliahou S., Kervaire M., Minimal resolutions of some monomial ideals, J. Algebra 129 (1) (1990), 1-25. MR 91b:13019

[4] Fatabbi G., On the resolution of ideals of fat points, J. Algebra 242 (2001), 92-108. MR2002d:13015

[5] Lyubeznik G., A new explicit finite resolution of ideals generated by monomials in an $R$ sequence, J. Pure Appl. Algebra 51 (1988), 193-195. MR89c:13020

[6] Taylor D., Ideals generated by monomials in an $R$-sequence, Thesis, University of Chicago, (1966).

Department of Mathematics, University of Genoa, Via Dodecaneso 35, 16146 Genoa, ITALY

E-mail address: valla@dima.unige.it 